Ich habe diesen speciellen Ausdruck so ausfihrlich abgeleitet, weil er nicht ganz mit dem von Laplace gegebenen übereinstimmt, auch wohl die Laplace'sche Aufstellung nicht leicht verständlich ist. Bei Laplace komnt nur das Integral vor, während das Glied $v_{1}$ fehlt. Dieser in praktischer Beziehung allerdings belanglose Fehler hat seinen Grund darin, dass a. a. O. die oben mit $z_{0}^{\prime}$ bezeichnete Integrationsgrenze von Anfang an durch $v_{1}$ ersetzt ist. Die Richtigkeit der Formel kann ubrigens auf einem völlig anderen Wege geprüft werden. Schiaparelli hat nämlich a. a. $O$. ein sehr schönes geometrisches Verfahren angegeben, welches die dufstellung von (6) wesentlich erleichtert und namentlich in dem Ealle, wo Rücksicht auf die Sonnenbewegung genommen wird, von Nutzen ist. Uebrigens wird dort die Endformel nicht abgeleitet.

Das Integral in (6) auszufüren macht nicht die geringsten Schwierigkeiten. Durch Differentiation des Ausdruckes

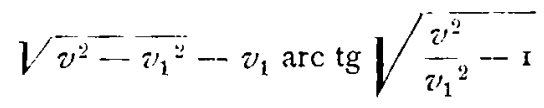

findet man sofort:

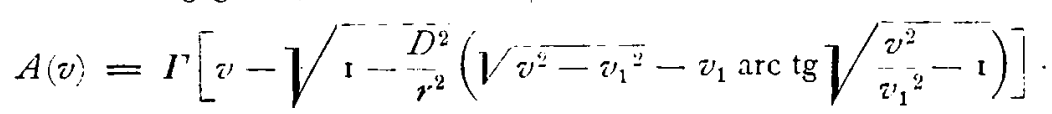

Für $v_{2}=\infty$ wird $A\left(v_{2}\right)=\infty$, also $W=0$; man hat daher in der That eine unendlich kleine Wahrscheinlichkeit für das Vorkommen nahe parabolischer Bahnen. Indessen nimmt $A(v)$ für mässige $v$ nur sehr langsam $z$, wie schon oben angedeutet. $Z u$ einem näheren Einblick in diese Verhältnisse ist der obige Ausdruck aber ebensowenig geeignet, wie er es zur numerischen Rechnung ist. Man wird indessen sehr leicht zu einer praktisch brauchbaren Entwickelung gelangen, wenn man bedenkt, dass $\frac{D}{r}$ sehr klein ist und Gleiches für die in Betracht kommenden Verhältnisse fur $v^{\prime}{ }_{1}$ gilt. Fur diese Anwendung genügt jedenfalls die folgende Annäherung

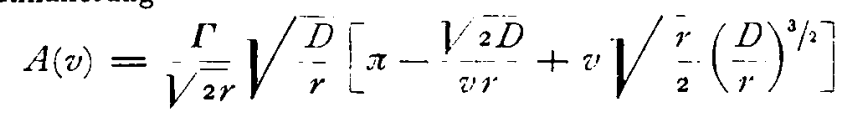

worin also nach dem obigen

$$
v^{2}=\frac{2}{r}-\frac{\mathrm{I}}{a}
$$

zu setzen ist. Fragt man nach der Wahrscheinlichkeit $W$ für das Vorkommen nur elliptisch und parabolisch gekrümmter Bahnen, so ist $a=\infty$ und $v \sqrt{r}=\sqrt{2}$ zu setzen. Die Einheit, in welcher $z_{2}$ anzusetzen ist, ist die Geschwindigkeit in der kreisförmigen Erdbahn. Mässig grosse Zahlen bedeuten demnach schon sehr bedeutende Geschwindigkeiten. Ich setze nun der Reihe nach $z_{2}=$ I, Io, 100. Ferner behalte ich die Zahlen des Laplace'schen Beispieles $D=2, r=100000$ bei, welche den thatsächlichen Verhältnissen, so gut dies eben geht, Rechnung tragen dürften. Er ergiebt sich dann der Reihe nach für $W$ :

$$
\text { I }-\frac{1}{703} ; \quad 1-\frac{1}{673} ; \quad 1-\frac{1}{489} \text {. }
$$

Das letzte Beispiel sagt aus, dass unter den gemachten Annahmen unter ungefähr 500 berechneten Cometenbahnen nach den Gesetzen der Wahrscheinlichkeit nur eine Bahn erwartet werden darf, welche ron einer Ellipse oder Parabel merklich abweicht. Hierbei ist vorausgesetzt, dass kosmische Ge. schwindigkeiten von $\circ$ bis zu etwa 400 geographischen Meilen pro Secunde gleich wahrscheinlich sind, eine Annahme, die gewiss sehr hoch gegriffen ist. Solch grosse relative Geschwindigkeiten $(\boldsymbol{v}=\mathbf{r} \circ \mathrm{o})$ sind bisher überhaupt noch nicht bemerkt worden und wir haben deshalb auch keinen Grund das Vorhandensein derselben zu berücksichtigen, wenn auch seine Möglichkeit selbstverständlich nicht in Abrede gestellt werden kann. Die grösste Gcschwindigkeit unter den Fixsternen hat vielleicht i 830 Groombridge. Nimmt man seine Parallaxe zu O". I und seine jährliche Gesammteigenbewegung zu 10", so ergiebt sich die Geschwindigkeit $v=16$. Ein in einer Parabel um die Sonne sich bewegender Comet hat die Maximalgeschwin. digkeit, wenn er die Sonnenoberfläche berührt, $z^{\prime}=2 \mathrm{I}$. Diese Zahlen beweisen natürlich gar nichts, sie dürften aber die Berechtigung enthalten, vorerst grössere Geschwindigkeiten, als oben angenommen, nur als Ausnahmefälle $z u$ betrachten. Im Uebrigen ist ersichtlich, dass die Zahlen unserer Beispiele noch mehr zu Gunsten des erlangten Resultates sprechen werden, wenn man $\varphi(\tau)$ so wählt, dass kleinere $v$ häufiger auftreten. Man kann dann, ohne principiell andere Resultate zu erhalten, die obere Grenze der möglichen Geschwindigkeiten bedeutend vergrössern

*) In einem im letzten Winter an hiesiger Liniversitüt abgehaltenen astronomischen Colloquium habe ich das Referat iiber Laplace's Arbeit Herrn Bodky übertragen. Derselbe wurde hierbei auf die Unvereinbarkeit der Laplace'schen Formel mit cier geometrischen Darstellung aufmerksam, wodurch ich die nöthige Correctur aufzusuchen veranlasst wurde. Herr Bodky hat übrigens die allgemeinere Aufgabe, welche die Eigenbewegung der Sonne etc. in Betracht zieht, in Angriff genommen.

\title{
Aufforderung betr. Beobachtungen des Cometen 1889 II.
}

Avendo impreso il calcolo degli elementi definitivi della cometa 1889 II, di cui corressi di già gli elementi (vedi A. N. 294I), bramerei di conoscere le osservazioni non ancora pubblicate nei periodici Astronomici.

Roma 1890 maggio 2.

E. Millosevich. 